\title{
Control of a Micro-Droplet for Laser-induced Breakdown Spectroscopy Solution Measurement
}

\author{
Satoshi Ikezawa ${ }^{1, a}$, Muneaki Wakamatsu ${ }^{1}$, Joanna Pawłat ${ }^{1}$ \\ and Toshitsugu Ueda ${ }^{1}$ \\ ${ }^{1}$ Graduate School of IPS, Waseda University. Hibikino 2-7-S257, Wakamatsu-ku, Kitakyushu-shi, \\ Fukuoka-ken, 808-0135, Japan \\ aikezawa@fuji.waseda.jp
}

Keywords: laser, LIBS, real-time measurement, micro-droplet

\begin{abstract}
In this paper, laser-induced breakdown spectroscopy (LIBS) using micro-droplet $\mathrm{NaCl}$ solution and set-up for control of micro-droplets are described. Micro-droplets controlling technique is important for solution quantitative analysis. In this study, micro-droplet ejection system for sampling is designed and presented. This micro-droplet ejection system enable a constant volume of the sample liquid to be obtained and it takes advantage of the liquid physical state; the density of the solution can be controlled accurately. The method presented here generates small droplets (diameter $30 \mu \mathrm{m}$ ) by confining the entire volume of the sample material in the laser beam spot area (minimum beam spot diameter: $53.2 \mu \mathrm{m}$ ) and separating it from its surroundings. Using this liquid micronizing method, improved sensitivities are obtained. The Advantage of LIBS is a useful method for determining the elemental composition of various materials regardless of their physical state (solid, liquid, or gas) and without any preprocessing; it is a type of atomic emission spectroscopy (AES). Despite the advantage of qualitative analysis, quantitative analysis is difficult because of sample and plasma fluctuations. Generating constant volume of micro-size sample and proper sample control technique contribute to LIBS quantitative analysis.
\end{abstract}

\section{Introduction}

In the LIBS technique, a high-energy laser pulse is focused on a sample to create plasma. Emissions from atoms and ions in the plasma are collected using lenses, guided toward a spectrograph, a streak camera, and analyzed by a computer. Some of the well-known AES methods for vaporization and excitation involve electrode arcs and sparks, inductively coupled plasma (ICP), direct-coupled plasma (DCP), and microwave-induced plasma (MIP). These methods typically require laboratory analytical facilities for specific use. AES-based methods for elemental analysis have an advantage: the capability to detect all kinds of elements or multielements simultaneously. In addition, since it requires only optical access to the sample, LIBS has many advantages: it facilitates real-time analysis and in situ analysis. LIBS has been investigated extensively to establish a method for the proper chemical analysis of specimens [1]-[3]. Although qualitative analysis results can be performed for a spectrum only by using a wavelength calibration reference, quantitative analysis can be carried out on the basis of several fundamental approaches using conventional methods that require many calibration processes $[4,5]$. In this study, the measurements are based on fundamental approaches for quantitative analysis (microsecond time-gated spectroscopy) and on the repetitive single spark for averaging the spectra from many shots. Optimizing the key experimental parameters - proper spark alignment, time-gate delay, and intensity gate width — allows the experimental determination of the detection limit. The intensities can be compared using standard atomic line references, and this method is called optical emission spectroscopy (OES) analysis. Another method is chemometric analysis and it based on the comparison of samples with known composition. This study is based on OES analysis that uses calibration curves obtained from the intensity calibration method. Our group has been developing the LIBS technique for quantitative analysis so that the LIBS system can be 
applied to various fields. A large amount of calibration reference data on the intensity, particle size, and air pressure has been obtained [6]-[8]. In addition to this reference data, in order to establish a practical calibration method for quantitative analysis in various environmental conditions, a LIBS solution concentration calibration technique has been developed by using a micro-droplet ejection system with the aim of performing absolute calibration. By using LIBS measurement, the sensitivity of this new method using a micro-droplet ejection system is compared to that of the conventional technique employing a bulk liquid cell.

\section{Experimental setup}

A schematic representation of the LIBS system using a $30-\mu \mathrm{m} \mathrm{NaCl}$ solution droplet is shown in Fig. 1. The Nd:YAG laser (Big Sky Laser; model: Ultra) was controlled with the delay pulse generator (Stanford Research System, Inc., Model DG-535). The trigger signal caused by laser was input to the first delay pulse generator. It provided the delay signal to the streak camera and the synchronized signal to the second pulse delay generator which provide the delay signal to the micro-droplet control module. The laser was operated at $1064 \mathrm{~nm}$ to generate a 50-mJ pulse with a width of $8 \mathrm{~ns}$ (FWHM). When used for ablation, the laser pulse was focused with a lens that had a focal length of $50 \mathrm{~mm}$, thereby yielding a power density of $10^{12} \mathrm{~W} / \mathrm{cm}^{2}$. The micro-droplet was released by using the dispenser head. Emissions from the laser-produced plasma were collected using additional lenses and they were then guided to a spectrograph (Chromex 250IS). Subsequently, the emitted light was dispersed using a diffraction grating with 1200 lines/mm and the electrical signal was recorded using a streak camera (Hamamatsu Photonics) with a time resolution of $10 \mathrm{ps}$ or greater. Finally, the signal was processed and stored in a computer. Fig. 2 shows the scheme of the micro-droplet subsystem. The sample solution was injected into the piezo head. The micro-droplet generating electric signal was synchronized with the LED strobe output. LED and CCD camera are placed on stages which are available for vertical-direction scanning. The region with a continuous stream of micro-droplets was covered during wind breaking for protection from the fluctuation of the droplet position. Emissions from the laser-produced plasma were collected using additional lenses and were then guided to a spectrograph.

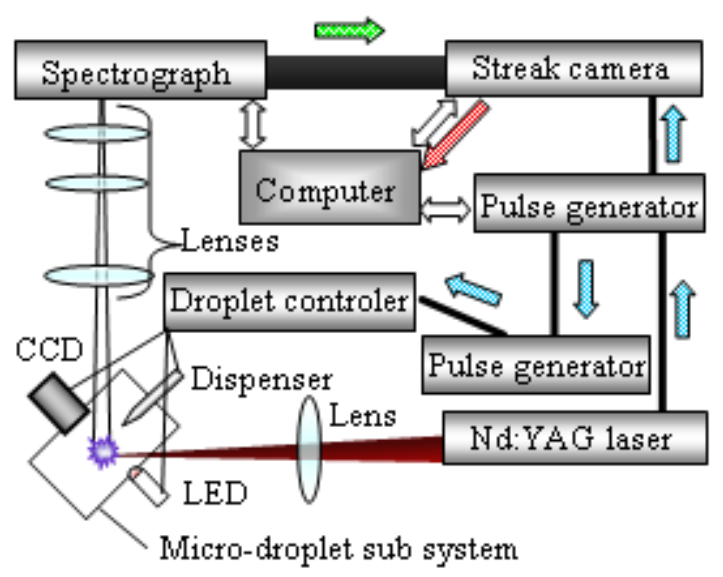

Fig. 1. Schematic representation of LIBS experiment using a 30- $\mu \mathrm{m}$ droplet ejection system 


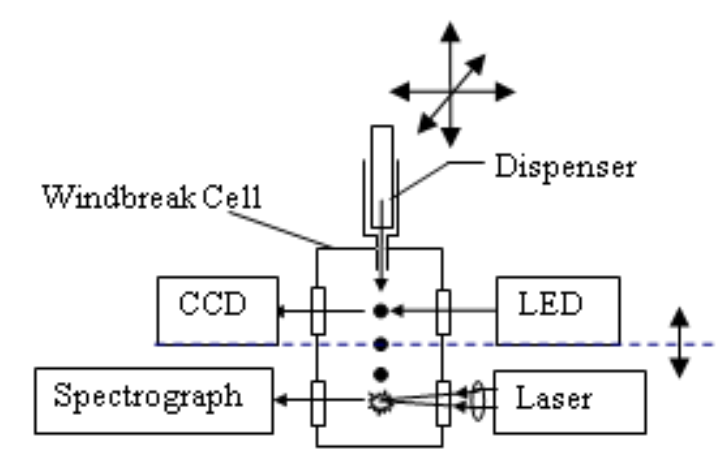

Fig. 2. Schematic representation of micro-droplet sub system

Fig. 3 shows the microphotograph of a micro-droplet ejection process. The micro-droplet nozzle ejected uniform droplets under stroboscopic illumination by an LED. The piezoelectric dispenser was capable of delivering up to 2000 droplets per second. The nozzle diameter was $30 \mu \mathrm{m}$. The droplet size could be varied in a narrow range by adjusting the voltage and voltage pulse duration. The velocity of the droplets increased with the voltage. A larger pulse duration led to larger droplets. In order to obtain the best stability and uniformity for the droplets, the optimum voltage parameters were set for every experiment. The piezoelectric nozzle was a droplet-on-demand device that provided single, isolated droplets with a diameter of $30 \mu \mathrm{m}$ and an initial velocity of $2 \mathrm{~m} / \mathrm{s}$. For the purpose of synchronizing the laser pulses with individual droplets, laser plasma was generated in a gaseous environment.

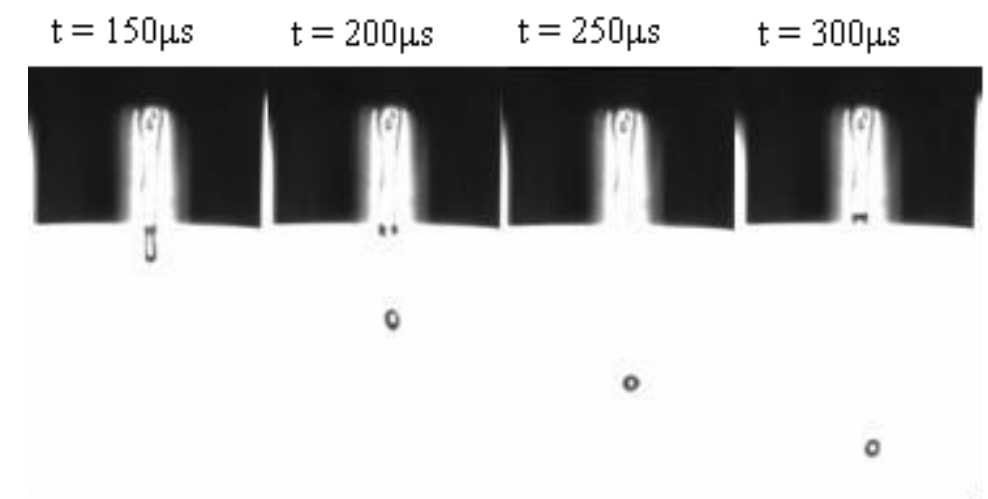

Fig. 3. Photograph of micro-droplet ejection from a nozzle with a diameter of $30 \mu \mathrm{m}$.

The photograph was taken under stroboscopic illumination at intervals of $50 \mu \mathrm{s}$ from $150 \mu$ s to $300 \mu$ s after trigger

\section{Results and discussion}

Figs. 4 to 6 show the experimental data for $0.1 \mathrm{M}$ to $0.3 \mathrm{M} \mathrm{NaCl}$ solution samples; these were obtained from a comparison between the LIBS spectral emission intensities obtained from the old bulk liquid method and those obtained from the $30-\mu \mathrm{m}$ droplet ejection system. An analysis based on data obtained from 100 laser pulse shots indicated that the central gate point was $18.1 \mu$ from trigger-in and the gate width was $3.9 \mu$ s. The analytical gate settings were optimized with $0.1 \mathrm{M} \mathrm{NaCl}$ solution. In Fig. 4, the comparison of data for the $0.1 \mathrm{M} \mathrm{NaCl}$ solution between the $30-\mu \mathrm{m}$ droplet ejection system and the bulk liquid method shows that the micro-droplet ejection system is more effective than the bulk liquid technique. From a comparison between the micro-droplet technique and the bulk liquid technique, it was observed that the data showed the advantage of using the new LIBS solution measuring technique. Figs. 5 and 6 show a comparison between the data for the $0.2 \mathrm{M} \mathrm{NaCl}$ solution and those for the $0.3 \mathrm{M} \mathrm{NaCl}$ solution. From these data, the atomic spectral intensity is observed to increase with the density of the solution. Similar to the case of the $0.1 \mathrm{M} \mathrm{NaCl}$ solution, 
the micro-droplet intensity is higher than the bulk liquid technique. By using the comparison data for $0.1 \mathrm{M}$ to $0.3 \mathrm{M} \mathrm{NaCl}$ Solution samples for this $30-\mu \mathrm{m}$ micro-droplet ejection system, Na calibration curves are shown in Fig. 7. The calibration curves for Na were plotted by using the average intensity of five measurements, each with 100 laser shots. It appears that the plasma conditions were different among these setups. In the case of the plasma in the bulk liquid system, the plasma created by the laser dissipated its energy to its surroundings or constituents. In the laser-created plasma plume, the distribution of atoms was such that the inner core of the plume, which mostly contained excited species, was surrounded by the unexcited species of the outer layer.

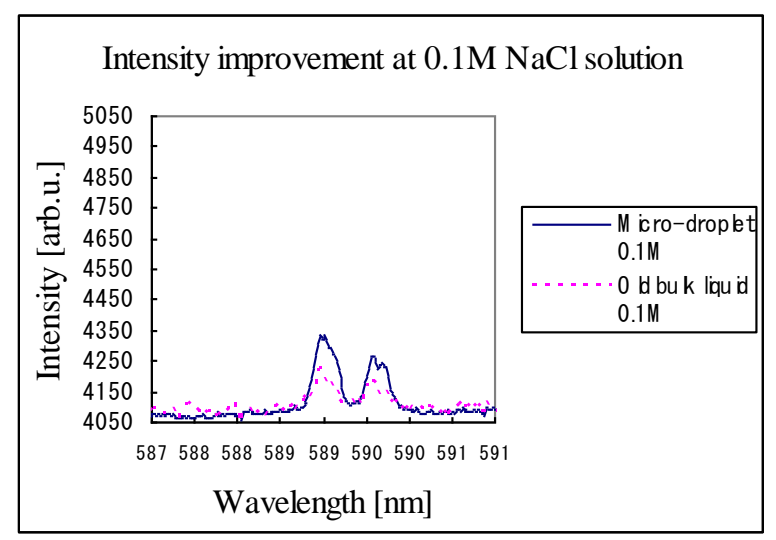

Fig. 4. Intensity difference for $0.1 \mathrm{M} \mathrm{NaCl}$ solution between bulk liquid measurement performed using the old technique and 30- $\mu \mathrm{m}$ micro-droplet measurement obtained from 100 laser pulse shots

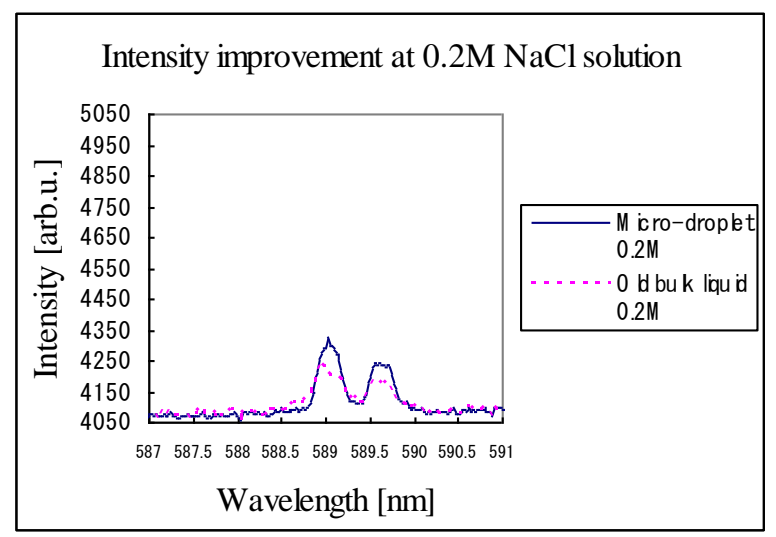

Fig. 5. Intensity difference for $0.2 \mathrm{M} \mathrm{NaCl}$ solution between bulk liquid measurement performed using the old technique and 30- $\mu \mathrm{m}$ micro-droplet measurement obtained from 100 laser pulse shots

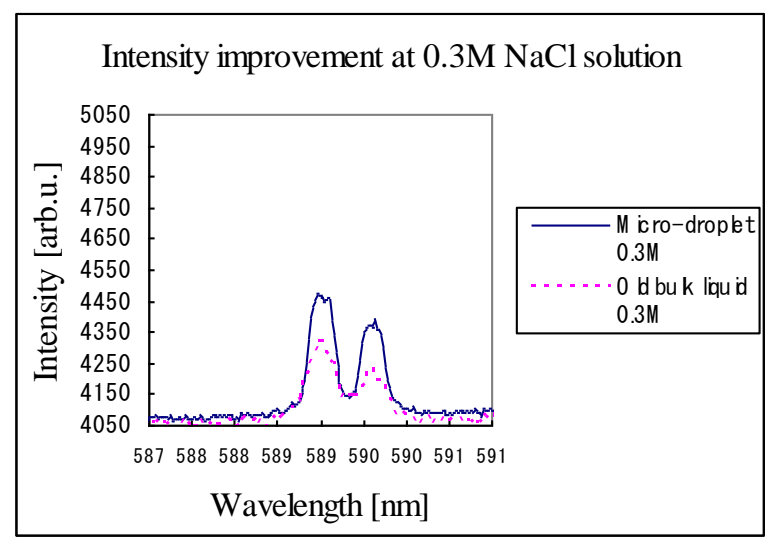

Fig. 6. Intensity difference for $0.3 \mathrm{M} \mathrm{NaCl}$ solution between bulk liquid measurement performed using the old technique and 30- $\mu \mathrm{m}$ micro-droplet measurement obtained from 100 laser pulse shots 


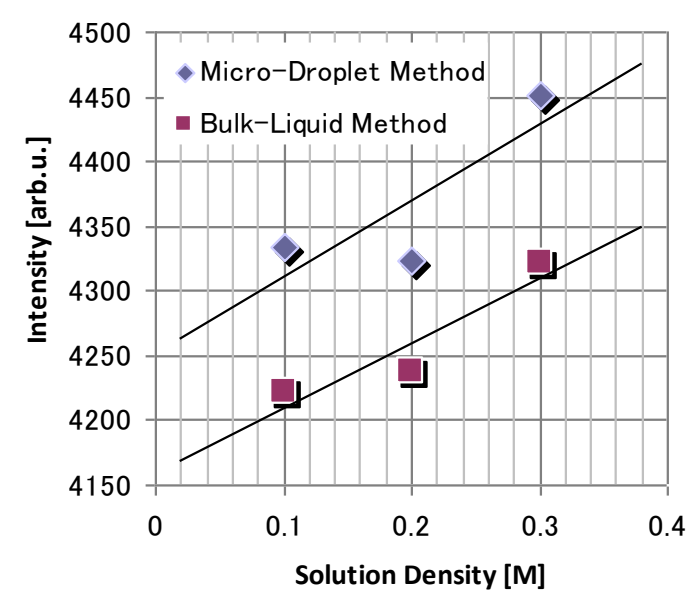

Fig. 7. Intensity improvement for $\mathrm{NaCl}$ solution measurements between old technique of bulk liquid method and 30- $\mu \mathrm{m}$ micro-droplet method obtained from 100 laser pulse shots

\section{Summary}

The new micro-droplet technique described here overcomes many of the disadvantages experienced with LIBS solution measurement. For previous methods, breakdowns on water surfaces produced strong emissions; however, the splashing of the sample at the water surface and chemical denaturation were practical and marked problems. For breakdowns passing through water, there was no splashing of the sample, but the emissions were very weak. In contrast, the new micro-droplet method described here, by micronizing the sample, made it possible for the entire volume of the liquid sample to be confined to the laser field and to be separated from the surroundings. This method was shown performance advantages of this method were strong emissions, absence of splashing of the sample, solution density controllability, and absence of chemical denaturation. The strong emission was attributed to the high energy transfer efficiency. In theoretical understanding, the irradiance threshold is governed by the cascade or avalanche growth of ionization, which is fed by absorption of the laser. Electrons in a beam field gain energy to ionize and increase in number through electron-neutral inverse bremsstrahlung. In the case of former method, in the bulk liquid, most of the laser energy was used for vaporizing the sample. In contrast, the use of the micro-droplet ejection system allowed most of the laser energy to be consumed by ionizing the sample. Therefore, different data were obtained from each LIBS technique and different calibration curves were required even for the same solution. We obtained the results that micro-droplet output system which could generate same volume of sample was employed in LIBS experiments. The technique needs to be developed further, but has the potential to overcome many of the problems associated with standard bulk-liquid-based techniques. Although precise measurements of the involved spatial and temporal beam parameter along the focal volumes remain difficult, the main processes using micro-droplet subsystem for liquid quantitative LIBS measurement have now emerged from a main feature of observations.

\section{References}

[1] H. A. Archontaki and S. R. Crouch: Evaluation of an Isolated Droplet Sample Introduction System for Laser-induced Breakdown Spectroscopy, Applied Spectroscopy, Volume 42, Number 5, 1988, pp.741-746.

[2] Christoph Janzen, Rüdiger Fleige, Reinhard Noll, Henrich Schwenke, Wilhelm Lahmann, Joachim Knoth, Peter Beaven, Eckard Jantzen, Andreas Oest, Peter Koke: Analysis of Small Droplets with a New Detector for Liquid Chromatography Based on Laser-induced Breakdown Spectroscopy, Spectrochimica Acta Part B, Volume 60, 2005, pp.993-1001. 
[3] Akshaya Kumar, Fang Y. Yueh, Tracy Miller and Jagdish P. Singh: Detection of Trace Elements in Liquids by Laser-induced Breakdown Spectroscopy with a Meinhard Nebulizer, Applied Optics, Volume 42, Number 30, 2003, pp.6040-6046.

[4] Liang Xu, Valery Bulatov, Vladimir V. Gridin and Israel Schechter: Absolute Analysis of Particulate Materials by Laser-induced Breakdown Spectroscopy, Analytical Chemistry, Volume 69, Number 11, 1997, pp.2103-2108.

[5] Susumu Nakamura, Yoshiro Ito, Kazuhiro Sone, Hitoshi Hiraga and Kenichi Kaneko: Determination of an Iron Suspension in Water by Laser-induced Breakdown Spectroscopy with Two Sequential Laser Pulses, Analytical Chemistry, Volume 68, Number 17, 1996, pp.2981-2986.

[6] Muneaki Wakamatsu and Toshitsugu Ueda: Particle Measurement Using LIBS, SICE Annual Conference 2005 in Okayama, 2005, pp.3717-3720.

[7] Alexander Andreev and Toshitsugu Ueda: Simulation of Laser Plasma Emission Characteristics of Small Solid Particles in Different Gas Atmospheres at Various Pressures, Trans. IEE of Japan, Volume 121-E, Number 11, 2001, pp.593-598.

[8] Muneaki Wakamatsu, Satoshi Ikezawa and Toshitsugu Ueda: Particle Element and Size Simultaneous Measurement Using LIBS, Trans. IEE of Japan, Volume 127-E, Number 9, 2007, pp.397-402. 